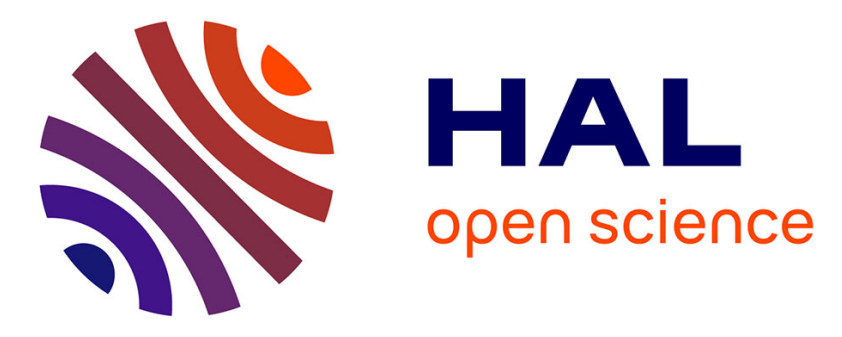

\title{
Perspectives dans la maladie de Gorham-Stout
}

C. Estublier, P. Chavassieux, E. Vignot, P. Guggenbuhl, C.B. Confavreux

\section{To cite this version:}

C. Estublier, P. Chavassieux, E. Vignot, P. Guggenbuhl, C.B. Confavreux. Perspectives dans la maladie de Gorham-Stout. Revue du Rhumatisme monographies, 2019, 86 (2), pp.126-131. 10.1016/j.monrhu.2018.12.003 . hal-02094605

\section{HAL Id: hal-02094605 \\ https://hal-univ-rennes1.archives-ouvertes.fr/hal-02094605}

Submitted on 23 May 2019

HAL is a multi-disciplinary open access archive for the deposit and dissemination of scientific research documents, whether they are published or not. The documents may come from teaching and research institutions in France or abroad, or from public or private research centers.
L'archive ouverte pluridisciplinaire HAL, est destinée au dépôt et à la diffusion de documents scientifiques de niveau recherche, publiés ou non, émanant des établissements d'enseignement et de recherche français ou étrangers, des laboratoires publics ou privés. 


\title{
Perspectives dans la Maladie de Gorham -Stout
}

\author{
New insights in Gorham-Stout disease
}

Charline Estublier ${ }^{1-2}$, Pascale Chavassieux², Emmanuelle Vignot ${ }^{1}$, Pascal Guggenbuhl ${ }^{3-4}$, Cyrille. B Confavreux ${ }^{1-2}$

${ }^{1}$ Service de Rhumatologie - Hospices Civils de Lyon, 69310, Pierre Bénite - France

2 INSERM UMR 1033-Lyos, Université de Lyon - 6900 Lyon France

${ }^{3}$ Service de Rhumatologie - Centre Hospitalier Universitaire de Rennes - 33000 Rennes France

${ }^{4}$ Institut NUMECAN - INSERM U 1241-INRA U 1341, Université de Rennes 1, Rennes, France

\section{Addresse de correspondance :}

Pr Cyrille B. Confavreux,

Service de Rhumatologie, Centre Hospitalier Lyon Sud

165, Chemin du Grand Revoyet

69310, Pierre Bénite - France

Email : cyrille.confavreux@chu-lyon.fr 


\section{RÉSUMÉ}

La maladie de Gorham-Stout appartient au groupe des malformations lymphatiques et se traduit par une prolifération de cellules lymphatiques sous l'influence de facteurs de croissance (VEGF) conduisant à un envahissement progressif de l'os. L'os disparaît peu à peu donnant typiquement un aspect d'os fantôme. L'enjeu est le risque fracturaire et ses conséquences ainsi que le risque de chylothorax potentiellement fatal. Les mécanismes physiopathologiques sont encore incomplets. Les traitements rapportés dans la littérature pour cette maladie exceptionnelle qui touche de l'enfant à l'adulte sont les bisphosphonates, la radiothérapie, la chirurgie, l'interféron alpha $2 \mathrm{~b}$ et les anti-angiogéniques dont le plus prometteur est le sunitinib.

\section{Highlights}

- * Maladie osseuse exceptionnelle

- * Prolifération de cellules vasculaires lymphatiques sous l'influence de facteurs de croissance

- * Ostéolyse massive non tumorale

- * Complications: fractures sévères, chylothorax et dyspnée

- * Traitement par bisphosphonates, chirurgie, radiothérapie, interféron, antiangiogénique type sunitinib (anti-VEGFR)

Mots clés : Os fantôme, lymphangiomatose, VEGFR, radiothérapie, bisphosphonates, IL6

\section{ABSTRACT}

The Gorham-Stout disease belongs to lymphatic abnormalities and correspond to a lymphatic endothelial cell proliferation under the stimulation of growth factors. This proliferation leads to a bone invasion and progressively to a typical vanishing bone aspect. Fracture risk and its consequences, chylothorax with sometimes fatal issue are the main complications of this disease. Physiopathological mechanisms remain poorly understood. Treaments of this rare bone disease, susceptible to affect every age from infant to adults, rely on bisphosphonates, radiotherapy, surgery, interferon alpha $2 \mathrm{~b}$ and anti-angiogenic drugs such as sunitinib.

Keywords : bone loss, lymphangiomatosis, VEGFR, radiotherapy, bisphosphonates, IL6

\section{Introduction}

La maladie de Gorham-Stout, du nom des deux médecins qui en firent la description en 1955 [1], est un classique de la rhumatologie... mais reste une maladie rarissime avec seulement un peu plus de 300 cas 
décrits dans la littérature depuis le premier rapport original de Jackson en 1838 [2]. Malgré une époque actuelle résolument scientifique et des moyens d'investigation considérables, cette maladie reste mal comprise probablement du fait de sa rareté pour l'étudier et progresser dans la thérapeutique, pourtant essentielle dans les formes sévères mettant en jeu le pronostic vital.

\section{Classification et physiopathologie}

La maladie de Gorham fait partie des malformations vasculaires. La dernière classification de 2014 de la Société Internationale d'Etude des Malformations Vasculaires (ISSVA) [3] la classe dans les malformations lymphatiques. Elle est donc clairement à distinguer des hémangiomes, prolifération du tissu vasculaire sanguin, qui peuvent être a) bénins (unique ou mulitiples), b) agressifs ou c) s'intégrer dans une angiomatose kystique avec des lésions hémangiomateuses osseuses et viscérales.

Pour l'instant, ce sont surtout les descriptions histologiques qui ont permis de comprendre mieux les mécanismes de la maladie de Gorham [4]. Il semblerait que l'os soit peu à peu envahi par une prolifération de cellules lymphatiques qui perforent le tissu osseux à partir des canalicules donnant, au microscope, un aspect de vaisseaux endothéliaux nombreux, dilatés et anarchiques. Ces cellules lymphatiques ne présentent pas d'atypie cellulaire. Les vaisseaux lymphatiques sont remplis de lymphe. II n'y a pas non plus d'infiltrat inflammatoire cellulaire associé. Le primum movens de la maladie de Gorham semble bien être lymphatique et on parle d'une anomalie de lymphangiogénèse. Les techniques d'immunohistochimie ont montré plusieurs marquages : du CD31/CD34 typiques des cellules endotheliales et du D2-40 pour les cellules endotheliales lymphatiques [5]. En effet, l'anticorps D2-40 reconnaît la podoplanine, une glycoproteine transmembranaire des cellules lymphatiques. Les cellules expriment aussi LYVE-1 qui est le récepteur 1 de l'acide hyaluronique des cellules endothéliales lymphatiques. Par ailleurs, il a été observé une expression du VEGF et de son récepteur VEGFR-3. On pense que le VEGF est le principal facteur de croissance qui est à l'origine de cette lymphangiogenèse incontrôlée. C'est pour cela que cette maladie n'est pas classée dans la famille des cancers mais qu'elle est plus à considérer comme un excès de facteurs de croissance à l'origine de cette prolifération de cellules lymphatiques endothéliales non tumorales.

Sous l'effet de cette prolifération, le tissu osseux disparaît peu à peu, donnant l'aspect classiquement décrit de " maladie des os fantômes ". II n'y a en effet aucun aspect de condensation en périphérie de la lésion et on en reste en radiographie à un os évanescent. Le mécanisme de l'ostéolyse reste très mystérieux. Les données histologiques sont contradictoires. Certains investigateurs ont rapporté des zones d'ostéolyse avec des ostéoclastes. D'autres, y compris les auteurs initiaux Gorham et Stout, ont rapporté qu'il n'y avait pas de hausse de l'activité ostéoclastique. II est probable qu'en fait, les auteurs 
aient regardé la maladie à différentes phases : phase active de résorption ou phase de stabilité. Une étude récente [6] chez la souris a montré que les cellules endothéliales lymphatiques de la maladie de Gorham, produisaient un excès de M-CSF à l'origine de l'augmentation de l'ostéoclastogenèse. II n'a pas été vu d'augmentation de RANKL. Ces auteurs ont par ailleurs mis au point un modèle de lésion de maladie de Gorham osseuse. À côté du M-CSF et du RANKL, d'autres facteurs peuvent stimuler l'ostéoclastogenèse et la résorption osseuse. Parmi eux on trouve les cytokines inflammatoires dont l'IL6 et le VEGF. De façon intéressante, certains auteurs ont dosé l'IL-6 et le VEGF aux différentes phases de la maladie. Ils ont montré que IL-6 et VEGF étaient nettement augmentés en phase active de la maladie par rapport à la phase de stabilité $[4,7-12]$. De plus, pour l'IL-6, les patients semblaient avoir même en phase de stabilité des valeurs plus élevées.

La figure 1, présente des coupes histologiques d'une biopsie iliaque non décalcifiée chez une femme de 21 ans. On observe une résorption osseuse massive avec des encoches de résorption profondes avec de nombreux ostéoclastes de très grande taille renfermant un grand nombre de noyaux (Fig 1A et B). Cette résorption s'observe au sein d'une zone de fibrose très dense avec de nombreuses cellules endothéliales lymphatiques. A proximité, sont présents des ilots de tissu ostéoïde épais recouverts d'ostéoblastes (Fig 1C) mais sans trouble de la minéralisation. La texture osseuse associe des zones lamellaires, normales et des zones d'os tissé (Fig 1D).

L'ensemble plaide donc pour une part de résorption active par les ostéoclastes, ceci est tout à fait en phase avec l'efficacité des biphosphonates. Un autre mécanisme d'ostéolyse ne peut être exclu cependant. L'inertie ostéoblastique n'est pas comprise à l'heure actuelle, d'autant plus que le VEGF-A devrait stimuler la fonction ostéoblastique. Plus d'études sont nécessaires pour progresser dans la compréhension de cette maladie. 


\section{Diagnostic de la maladie}

\subsection{Terrain.}

La maladie de Gorham est possible à tout âge. Cependant la maladie se révèle habituellement chez l'enfant ou le jeune adulte. L'âge moyen au diagnostic est de 25 ans. Dans la littérature, il n'est pas discerné de sexe ratio particulier. II s'agit d'une maladie qui reste sporadique, sans transmission évidente d'un individu à l'autre par contagion ou par transmission génétique. Il est possible qu'il existe une part génétique à cette maladie, mais elle serait alors de type mutation ponctuelle mosaïque somatique. Les sociétés savantes spécialisées dans la maladie de Gorham (la Lymphatic Malformation Institute (LMI) et la Lymphangiomatosis Gorham Disease Alliance (LGDA)) recommandent de réaliser lors des futures biopsies un prélèvement de tissu pathologique non fixé non décalcifié en vue de l'analyse whole-exome du génome des tissus malades et identifier d'éventuelles mutations.

\subsection{Topographie.}

La maladie de Gorham peut toucher tous les os du corps. Il existe cependant, d'après les cas rapportés, une prédilection pour la mandibule, le maxillaire, les clavicules, les côtes, les vertèbres cervicales, le bassin (cadre obturateur) et le fémur. Classiquement, la maladie touche un os ou plusieurs os de façon contiguë. Sur les 200 cas rapportés environ, 50 touchaient le massif maxillofacial [13].

\subsection{Sémiologie.}

Au plan fonctionnel, la maladie peut être longtemps asymptomatique jusqu'à ce qu'elle se révèle par des douleurs localisées permanentes, une faiblesse ou par des complications. Au niveau osseux la complication typique est la fracture de fragilité, consécutive à un traumatisme mineur voire sans traumatisme, dont les conséquences dépendent de la pièce osseuse touchée. Lorsque l'atteinte est au niveau d'un os long, il peut s'agir de fracture avec impotence fonctionnelle importante. Lorsqu'il s'agit d'une atteinte vertébrale, il y a un risque de déformation majeure (cyphose, séquelles douloureuses mécaniques, préjudice esthétique), mais également un risque neurologique majeur avec suivant les étages compression médullaire, paralysie ou décès comme ce fut le cas pour la patiente présentée en figure 2. Elle souffrait d'une atteinte occipitale majeure inopérable et est décédée par compression du tronc cérébral. En dehors de l'aspect rhumatologique osseux, l'autre complication majeure de la maladie de Gorham est l'accumulation de lymphe au niveau thoracique. Le chylothorax est parfois révélateur de la maladie sous forme d'une dyspnée. Il peut être source de détresse respiratoire engageant le pronostic vital de ces patients [7]. On estime que $25 \%$ des patients présentant une 
maladie de Gorham sont à risque de développer un chylothorax [14]. Certains patients présentent des épanchements sous cutanés de lymphe. Sur le plan évolutif, il est totalement inconnu pour l'instant pourquoi à certains moments la maladie est en phase active et progressive et pourquoi à d'autres elle se stabilise. De même sur le déclenchement, certains ont évoqué un traumatisme mineur mais ceci n'est qu'observationnel et non constant.

\subsection{Imagerie.}

Au plan radiologique, la maladie se présente comme une ostéolyse agressive initialement localisée qui progressivement s'agrandit jusqu'à faire disparaître littéralement l'ensemble de la pièce osseuse voire plusieurs pièces osseuses en contiguïté. Cette ostéolyse ne présente pas de condensation périphérique. Certaines images sont très impressionnantes comme la disparition complète de l'hémibassin droit d'une femme au cours de 14 ans de suivi rapportée par Ballas [15]. Les parties molles adjacentes peuvent être infiltrées par la lésion. L'aspect classique des os long est décrit en " bâton de sucette » [16]. Le scanner osseux est l'examen complémentaire de choix pour évaluer avec précision cette ostéolyse. En I.R.M., l'aspect est souvent fortement vascularisé. L'importance du rehaussement n'a pas été corrélée à l'activité de la maladie. L'I.R.M. permet également d'évaluer l'atteinte des parties molles adjacentes. Le cas de M. E, 53 ans, illustre parfaitement une ostéolyse diffuse qui s'est révélée par des douleurs osseuses depuis l'âge de 28 ans. II présente également des anévrysmes artério-veineux multiples. Les principales lésions osseuses se situent sur le rachis lombaire, le bassin, les cols fémoraux mais également les genoux et les pieds. De façon intéressante, on observe en lieu et place de l'os des calcifications ponctuelles dans les parties molles. L'IRM du bassin montre également de nombreuses ectasies vasculaires de la loge des fessiers prédominant à droite (figure 3).

\subsection{Biologie.}

Le bilan biologique est pauvre et sans aide pour le diagnostic positif. Il est par contre essentiel pour le diagnostic différentiel d'une hémopathie et des autres causes d'ostéolyse. II n'est pas retrouvé de syndrome inflammatoire. La numération, l'électrophorèse des protéines plasmatiques et les LDH sont normales. Le bilan phosphocalcique est habituellement normal. II n'est pas retrouvé non plus d'anomalie du remodelage osseux. II n'y a pas d’anomalie de la fonction rénale ou hépatique.

\subsection{Maladie apparentée.}

La frontière avec la lymphangiomatose également appelée malformation lymphatique généralisée (Generalized Lymphatic Anomaly GLA) est ténue. Cette maladie est également caractérisée par la 
prolifération de vaisseaux lymphatiques. Elle peut également toucher l'os. L'analyse américaine rétrospective comparative de 19 patients avec une maladie de Gorham et 32 avec une lymphangiomatose a montré que l'atteinte osseuse était légèrement différente. La maladie de Gorham est plutôt responsable d'une atteinte plus focalisée et l'ostéolyse progressive conduit à l'atteinte de l'os corticale. Typiquement, dans la lymphangiomatose, les lésions osseuses sont plus nombreuses mais restent confinées à la cavité médullaire et touchent volontiers le squelette appendiculaire. Il y a également plus de malformations kystiques systémiques associées dans la lymphangiomatose. Sur le plan histologique par contre il y a peu de différences [17].

\section{Diagnostic différentiel}

Il s'agit d'un diagnostic d'exclusion et l'ensemble des étiologies classiques d'ostéolyse doivent être écartées (infectieuses, tumorales, inflammatoires, endocriniennes, ostéolyse post traumatique pseudomaligne ...). Le cas de Mme C. âgée de 71 ans, illustre parfaitement la difficulté diagnostique en plus du risque neurologique de la maladie de Gorham. La patiente a présenté en 1987 un cancer du sein étiqueté métastatique avec une lésion ostéolytique de C1. La lésion a été retirée avec mise en place d'une ostéosynthèse postérieure. En 2006, les cervicalgies réapparaissent. L'imagerie met en évidence une ostéolyse de C2 et de l'occiput (figure 2). L'épisode a été interprété comme un granulome de résorption lié au matériel qui a donc été retiré. L’histologie a écarté une récidive métastatique et une histiocytose liée à I'X grâce aux immunomarquages. Le diagnostic de maladie de Gorham-Stout des 3 premières vertèbres cervicales, de la base et de la voûte du crâne a été posé. La maladie a continué d'évoluer vers une issue fatale par brusque compression du tronc cérébral.

\section{Prise en charge thérapeutique}

À I'heure actuelle, il n'existe aucun consensus thérapeutique, en l'absence d'essai clinique ayant permis d'évaluer l'efficacité d'un traitement. Les thérapeutiques utilisées reposent sur des données physiopathologiques et sur l'expérience rapportée dans la littérature. Le traitement de la maladie de Gorham dépend du siège des pièces osseuses impliquées et de la sévérité de la maladie. Plusieurs volets ont été essayés : la chirurgie orthopédique, la radiothérapie, différents traitements médicaux dont l'interféron alfa-2b, les biphosphonates, les anti-angiogéniques et la chimiothérapie. Cette prise en charge a pour objectif de stabiliser la maladie. Elle doit être associée suivant les cas à des traitements de support dont nous ne parlerons pas ici comme la ventilation assistée en réanimation pour les chylothorax, la neurochirurgie en cas de compression médullaire, les anti-douleurs ... 


\subsection{Traitement local.}

La chirurgie seule a été utilisée à plusieurs reprises pour stabiliser les régions impliquées. Les résultats de la chirurgie seule sont très mitigés avec de nombreuses rechutes de la maladie. Ellati dans sa synthèse souligne que sur 26 cas opérés soit par résection simple soit par résection-prothèse soit par résection puis reconstruction avec greffe osseuse, seuls 11 patients ont été contrôlés [18]. Il y a aussi de nombreux perdus de vue, ce qui fragilise l'analyse. Sur cette base, il a proposé une radiothérapie adjuvante à la chirurgie sous forme de 40 Gy à raison de 2 Gy par fraction avec un bon résultat dans le cas rapporté. En outre, il a complété son traitement local d'un traitement systémique par biphosphonate. Dans la mesure du possible, on évitera la chirurgie seule. D'autres ont utilisé la radiothérapie seule avec une dose totale de 36 à 45 Gy par fraction de 2 Gy [4,19-23]. Cette attitude donne plutôt de bons résultats mais est limite dans son indication vu le caractère non tumoral de la lésion et le risque de complications secondaires à ces doses, surtout chez des enfants, notamment le risque d'angiosarcome induit. Tout est affaire de balance bénéfice-risque.

\subsection{Traitement systémique.}

L'approche médicale permet dans un certain nombre de cas de stabiliser la maladie. Les traitements qui ont été utilisés dans la littérature sont l'interferon alpha 2b [24-26] et les biphosphonates par voie orale (clodronate, alendronate) ou veineuse (pamidronate, acide zolédronique) [11,26,27]. II est très difficile d'avoir une idée claire de l'attitude à adopter vu l'hétérogénéité des schémas dans la littérature, la disparité d'âge (pédiatrie, adolescent, adulte jeune, adulte) et les variations de sévérité (forme osseuse, chylothorax). II n'est pas rare non plus d'observer des combinaisons thérapeutiques (interferon et bisphosphonate par exemple).

Une initiative intéressante a été proposée par Rössler et al. sur la base des données de l'immunohistochimie et du marquage vasculaire [28]. Rössler a montré l'intérêt de traiter par un antiVEGFR, le sunitinib en association avec du taxol à faible dose métronomique $\left(10 \mathrm{mg} / \mathrm{m}^{2} / \mathrm{semaine}\right)$. La justification du taxol est qu'il s'agit d'un inhibiteur de tubuline qui vient donc bloquer l'expansion des cellules endothéliales lymphatiques même si elles ne sont pas tumorales en tant que tel. La stratégie a été utilisée chez deux enfants de 7 et 14 ans, atteints respectivement d'une forme sévère de lymphangiomatose et de maladie de Gorham avec chylothorax. Le patient atteint de GLA était déjà en échec de chimiothérapie conventionnelle. Dans les deux cas, les patients avaient en plus un régime pauvre en triglycérides à chaîne moyenne. Le sunitinib trouvait aussi sa justification dans son action bloquante également du PDGFR déjà impliqué auparavant dans la maladie de Gorham [29]. 
D'autres thérapeutiques comme des inhibiteurs de la rapamycine sont à l'étude mais pour l'instant la prise en charge des patients atteints de maladie de Gorham est loin d'être codifiée. Elle représente cependant un enjeu important pour cette maladie rare mais potentiellement très sévère voire fatale.

\section{REMERCIEMENTS}

Les auteurs remercient Nadege Trehet-Mendel pour son aide dans la requête informatique des cas.

Déclaration de liens d'intérêt : Les auteurs ne déclarent pas de conflits pour ce travail. 


\section{Références}

[1] Gorham LW, Stout AP. Massive osteolysis (acute spontaneous absorption of bone, phantom bone, disappearing bone); its relation to hemangiomatosis. J Bone Joint Surg Am 1955;37-A:985-1004.

[2] Jackson J. A boneless arm. Boston Med Surg J 1838:368-9.

[3] Wassef M, Blei F, Adams D, et al. Vascular Anomalies Classification: Recommendations From the International Society for the Study of Vascular Anomalies. Pediatrics 2015;136:e203-214.

[4] Dellinger MT, Garg N, Olsen BR. Viewpoints on vessels and vanishing bones in Gorham-Stout disease. Bone 2014;63:47-52.

[5] Liu Y, Zhong D-R, Zhou P-R, et al. Gorham-Stout disease: radiological, histological, and clinical features of 12 cases and review of literature. Clin Rheumatol 2016;35:813-23.

[6] Wang W, Wang H, Zhou X, et al. Lymphatic Endothelial Cells Produce M-CSF, Causing Massive Bone Loss in Mice. J Bone Miner Res Off J Am Soc Bone Miner Res 2017;32:939-50.

[7] Fujiu K, Kanno R, Suzuki H, et a. Chylothorax associated with massive osteolysis (Gorham's syndrome). Ann Thorac Surg 2002;73:1956-7.

[8] Brodszki N, Länsberg J-K, Dictor M, et al. A novel treatment approach for paediatric Gorham-Stout syndrome with chylothorax. Acta Paediatr Oslo Nor 1992 2011;100:1448-53.

[9] Morimoto N, Ogiwara H, Miyazaki O, et al. Gorham-Stout syndrome affecting the temporal bone with cerebrospinal fluid leakage. Int J Pediatr Otorhinolaryngol 2013;77:1596-600.

[10] Dupond J-L, Bermont L, Runge $M$, et al. Plasma VEGF determination in disseminated lymphangiomatosis-Gorham-Stout syndrome: a marker of activity? A case report with a 5-year follow-up. Bone 2010;46:873-6.

[11] Hammer F, Kenn W, Wesselmann U, et al. Gorham-Stout disease--stabilization during bisphosphonate treatment. J Bone Miner Res Off J Am Soc Bone Miner Res 2005;20:350-3.

[12] Devlin RD, Bone HG, Roodman GD. Interleukin-6: a potential mediator of the massive osteolysis in patients with Gorham-Stout disease. J Clin Endocrinol Metab 1996;81:1893-7.

[13] Naqvi AA, Joshi SS, Bailey E. An unusual case of disappearing bone disease in the mandible and literature review. J Surg Case Rep 2017;2017:rjx025.

[14] Tie ML, Poland GA, Rosenow EC. Chylothorax in Gorham's syndrome. A common complication of a rare disease. Chest 1994;105:208-13.

[15] Ballas R, Caduc M, Ovigue J, et al. Fourteen years follow-up of massive pelvic girdle osteolysis caused by lymphatic malformation (Gorham-Stout Disease). Jt Bone Spine Rev Rhum 2017;84:625.

[16] Yerganyan VV, Body JJ, De Saint Aubain N, et al. Gorham-Stout disease of the proximal fibula treated with radiotherapy and zoledronic acid. J Bone Oncol 2015;4:42-6. 
[17] Lala S, Mulliken JB, Alomari Al, et al. Gorham-Stout disease and generalized lymphatic anomaly-clinical, radiologic, and histologic differentiation. Skeletal Radiol 2013;42:917-24.

[18] Ellati R, Attili A, Haddad H, et al. Novel approach of treating Gorham-Stout disease in the humerus-Case report and review of literature. Eur Rev Med Pharmacol Sci 2016;20:426-32.

[19] Heyd R, Micke O, Surholt C, et al. Radiation therapy for Gorham-Stout syndrome: results of a national patterns-of-care study and literature review. Int J Radiat Oncol Biol Phys 2011;81:e179-185.

[20] Dunbar SF, Rosenberg A, Mankin H, et al. Gorham's massive osteolysis: the role of radiation therapy and a review of the literature. Int J Radiat Oncol Biol Phys 1993;26:491-7.

[21] Duffy BM, Manon R, Patel RR, et al. A case of Gorham's disease with chylothorax treated curatively with radiation therapy. Clin Med Res 2005;3:83-6.

[22] Fontanesi J. Radiation therapy in the treatment of Gorham disease. J Pediatr Hematol Oncol 2003;25:816-7.

[23] McNeil KD, Fong KM, Walker QJ, et al. Gorham's syndrome: a usually fatal cause of pleural effusion treated successfully with radiotherapy. Thorax 1996;51:1275-6.

[24] Takahashi A, Ogawa C, Kanazawa T, et al. Remission induced by interferon alfa in a patient with massive osteolysis and extension of lymph-hemangiomatosis: a severe case of Gorham-Stout syndrome. J Pediatr Surg 2005;40:E47-50.

[25] Leite I, Hernández-Martín A, Colmenero I, et al. Invasive lymphatic malformation (gorham-stout) of the pelvis with prominent skin involvement. Pediatr Dermatol 2013;30:374-8.

[26] Kuriyama DK, McElligott SC, Glaser DW, et al. Treatment of Gorham-Stout disease with zoledronic acid and interferon- $\alpha$ : a case report and literature review. J Pediatr Hematol Oncol 2010;32:579-84.

[27] Avelar RL, Martins VB, Antunes AA, et al. Use of zoledronic acid in the treatment of Gorham's disease. Int J Pediatr Otorhinolaryngol 2010;74:319-22.

[28] Rössler J, Saueressig U, Kayser G, et al. Personalized Therapy for Generalized Lymphatic Anomaly/Gorham-Stout Disease With a Combination of Sunitinib and Taxol. J Pediatr Hematol Oncol 2015;37:e481-485.

[29] Hagendoorn J, Padera TP, Yock TI, et al. Platelet-derived growth factor receptor-beta in Gorham's disease. Nat Clin Pract Oncol 2006;3:693-7. 


\section{Figure 1 :}

Coupes histologiques d'une biopsie iliaque non décalcifié. A-B : Surfaces de résorption très étendues et profondes renfermant de très nombreux et volumineux ostéoclastes multinucléés $(*)$. Cette résorption s'observe au sein d'une zone de fibrose très dense avec de nombreuses cellules endothéliales lymphatiques. C : Présence d'ilots de tissu ostéoïde bordés d'ostéoblastes actifs ( $\rightarrow$ ). D : Zone d'os tissé (OT) bordant une zone de tissu osseux lamellaire. A-B-C : trichrome de Goldner. D : solochrome cyanine R. Grossissements : x50 (D), x100 (A et C), x200 (B).

\section{Figure 2 :}

Cas d'une patiente de 71 ans qui présente une maladie de Gorham-Stout avec ostéolyse massive de l'occiput bien visible sur la radiographie simple (A). Les images B,C,D sont des coupes coronales de scanner en fenêtres osseuses montrant l'atteinte majeure de l'occiput mais aussi des vertèbres cervicales C1 et C2 exposant au risque de compression du tronc cérébral.

\section{Figure 3 :}

Cas d'un patient de 53 ans présentant une maladie de Gorham-Stout avec ostéolyse massive multifocale. Les images A (radiographie simple) et B (scanner osseux en coupes frontales osseuses) montrent une ostéolyse du col fémoral gauche, de la sacro-iliaque droite et des calcifications de remplacement au-dessus du col fémoral droit. Les images $C$ et $D$, respectivement en radiographie standard et en scanner, montrent l'atteinte du pied. L'image E montre la disparition en scanner du corps postérieur droit de la vertèbre L4. L'image F présente en IRM pondérée en T1 de multiples ectasies vasculaires de la loge des fessiers droits essentiellement. Il existe également quelques ectasies à gauche. 


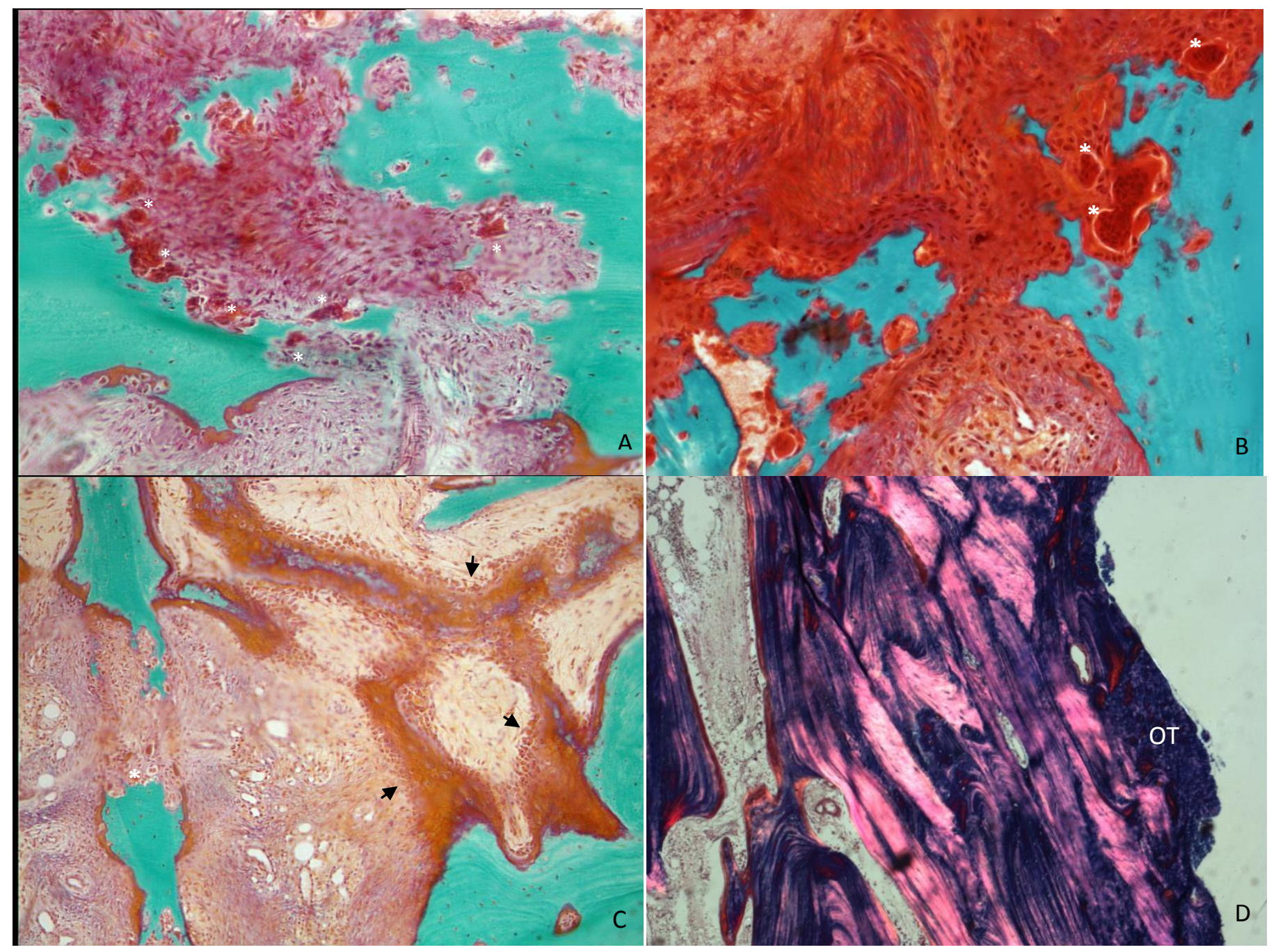

Figure 1. Coupes histologique d'une biopsie iliaque non décalcifié. A-B: Surfaces de résorption très étendues et profondes renfermant de très nombreux et volumineux ostéoclastes multinucléés $\left({ }^{*}\right)$. Cette résorption s'observe au sein d'une zone de fibrose très dense avec de nombreuses cellules endothéliales lymphatiques. C: Présence d'ilots de tissu ostéoïde bordés d'ostéoblastes actifs $(\rightarrow)$. D: Zone d'os tissé (OT) bordant une zone de tissu osseux lamellaire. A-B-C : trichrome de Goldner. D: solochrome cyanine R. Grossissements: x50 (D), x100 (A et C), x200 (B). 

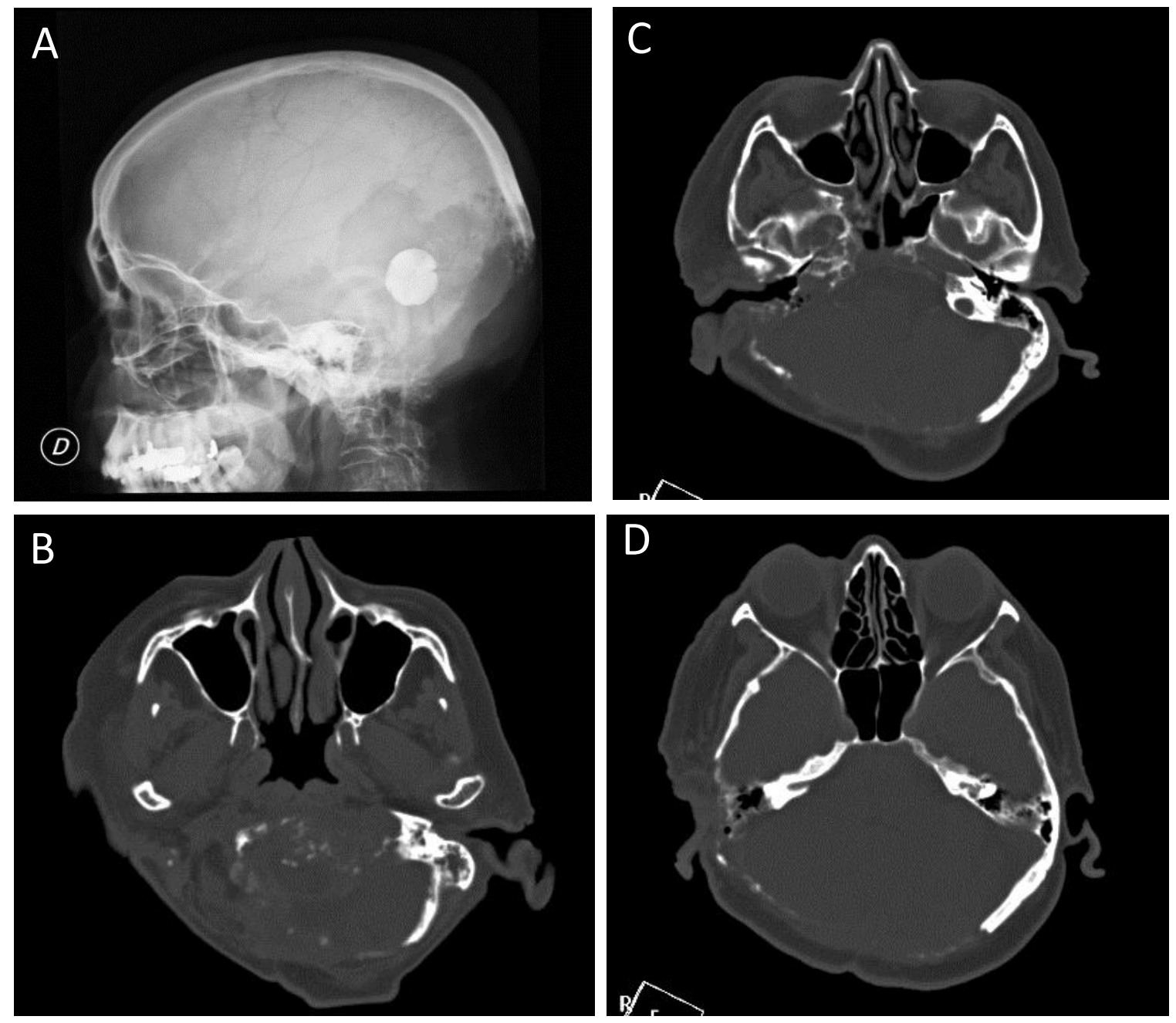

Figure 2

Cas d'une patiente de 71 ans qui présente une maladie de Gorham-Stout avec ostéolyse massive de l'occiput bien visible sur la radiographie simple (A). Les images B,C,D sont des coupes coronales de scanner en fenêtres osseuses montrant l'atteinte majeure de l'occiput mais aussi des vertèbres cervicales $\mathrm{C} 1$ et $\mathrm{C} 2$ exposant au risque de compression du tronc cérébral. 

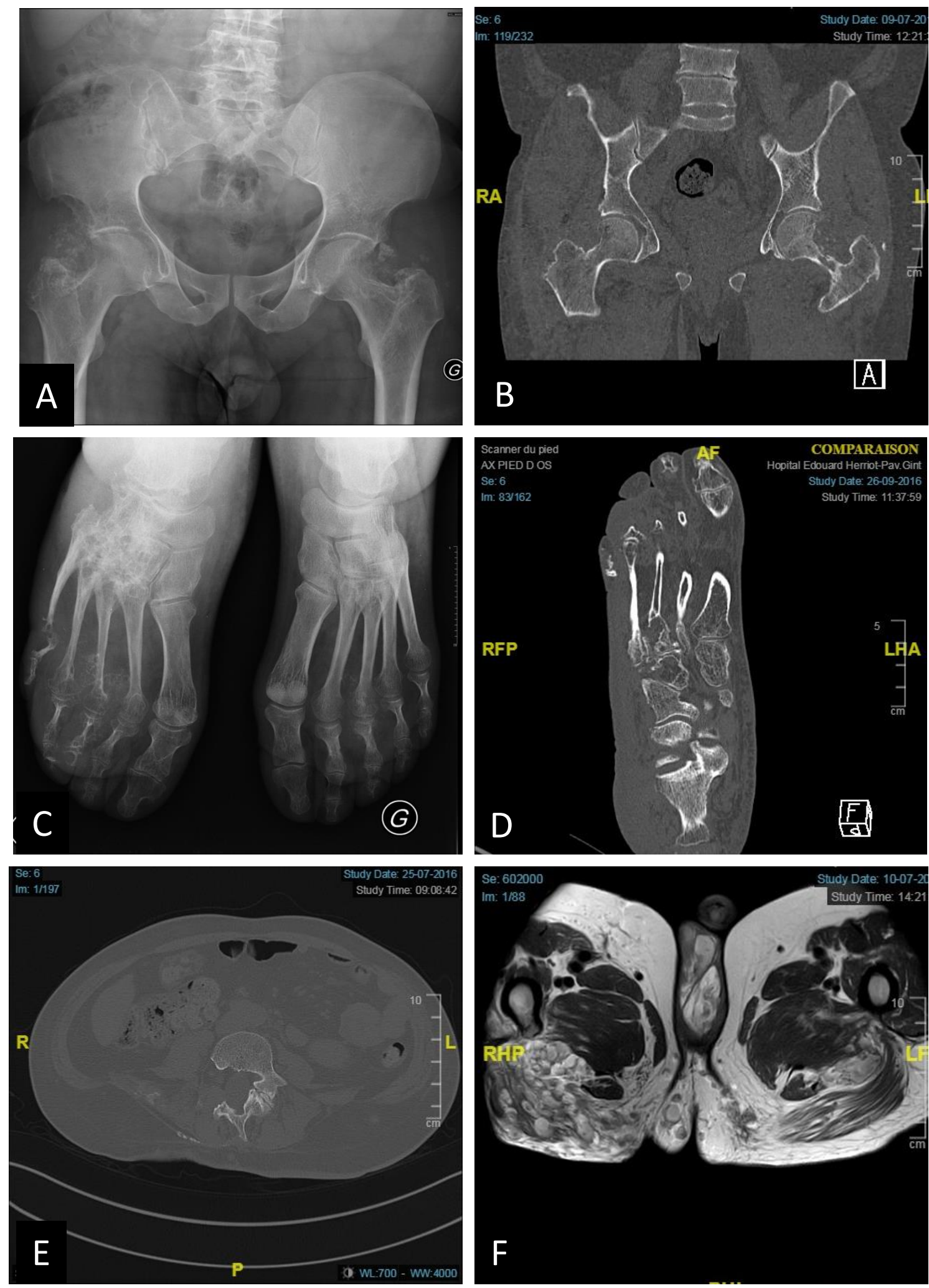

Figure 3: Cas d'un patient de 53 ans présentant une maladie de Gorham-Stout avec ostéolyse massive multifocale. Les images $A$ (radiographie simple) et B (scanner osseux en coupes frontales osseuses) montrent une ostéolyse du col fémoral gauche, de la sacroiliaque droite et des calcifications de remplacement au-dessus du col fémoral droit. Les images $C$ et $D$, respectivement en radiographie standard et en scanner, montrent l'atteinte du pied. L'image $E$ montre la disparition en scanner du corps postérieur droit de la vertèbre L4. L'image F présente en IRM pondérée en T1 de multiples ectasies vasculaires de la loge des fessiers droits essentiellement. II existe également quelques ectasies à gauche. 